Article

\title{
A Compact Double-Folded Substrate Integrated Waveguide Re-Entrant Cavity for Highly Sensitive Humidity Sensing
}

\author{
Zhihua Wei, Jie Huang *, Jing Li, Junshan Li, Xuyang Liu and Xingsheng Ni \\ College of Engineering and Technology, Southwest University, Chongqing 400715, China \\ * Correspondence: jiehuang@swu.edu.cn; Tel.: +86-139-9643-0948
}

Received: 22 June 2019; Accepted: 25 July 2019; Published: 27 July 2019

check for updates

\begin{abstract}
In this study, an ultra-compact humidity sensor based on a double-folded substrate integrated waveguide (SIW) re-entrant cavity was proposed and analyzed. By folding a circular re-entrant cavity twice along its two orthogonally symmetric planes, the designed structure achieved a remarkable size reduction (up to $85.9 \%$ ) in comparison with a conventional $\mathrm{TM}_{010}$-mode circular SIW cavity. The operating principle of the humidity sensor is based on the resonant method, in other words, it utilizes the resonant properties of the sensor as signatures to detect the humidity condition of the ambient environment. To this end, a mathematical model quantitatively relating the resonant frequency of the sensor and the relative humidity $(\mathrm{RH})$ level was established according to the cavity perturbation theory. The sensing performance of the sensor was experimentally validated in a $\mathrm{RH}$ range of $30 \%-80 \%$ by using a humidity chamber. The measured absolute sensitivity of the sensor was calculated to be $135.6 \mathrm{kHz} / \% \mathrm{RH}$, and the corresponding normalized sensitivity was $0.00627 \% / \%$ RH. It was demonstrated that our proposed sensor not only has the merits of compact size and high sensitivity, but also benefits from a high Q-factor and ease of fabrication and integration. These advantages make it an excellent candidate for humidity sensing applications in various fields such as the agricultural, pharmaceutical, and food industries.
\end{abstract}

Keywords: microwave; humidity sensor; folded SIW; re-entrant cavity resonator

\section{Introduction}

Humidity is one of the most important indexes for evaluating environmental quality, and it is crucial to monitor and control humidity accurately in many scenarios. For instance, the relative humidity $(\mathrm{RH})$ level in greenhouses is a key factor that affects the growth and disease incidence of crops [1]. For food storage, the RH needs to be controlled at a proper level so as to prevent the putridity of foods [2]. Other fields such as material processing, industrial manufacturing, as well as electrical equipment operating also have rigorous requirements on the $\mathrm{RH}$ of the environment [3,4]. Within this context, developing humidity sensors with high performance has aroused considerable enthusiasm and become a subject of intensive research.

Traditional humidity sensors involve various types including resistive [5-7], capacitive [8-10], quartz crystal microbalance (QCM) [11,12], piezoelectric [13], and so on. Most of these sensors, however, operate at very low frequencies (quasi-direct current (DC)). This makes them difficult to be utilized directly in modern radio frequency (RF) sensing and detection systems. Fortunately, the passive microwave sensor has been recently exploited and developed as a promising alternative for $\mathrm{RH}$ detection. The passive microwave sensor can not only be conveniently applied to RF sensing systems, but also offers the merits of low cost, high design flexibility, and freedom from a power supply. Thus far, a number of passive microwave RH sensing schemes have been reported [14-26]. One branch of 
this research concentrates on the synthesis of novel sensing materials and their combinations with diverse microwave components; it is dedicated to improving the sensing performance of sensors (e.g., sensitivity, resolution, etc.). A variety of sensing materials such as polyvinyl-alcohol (PVA) [14], poly (3,4-ethylenedioxythiophene): poly (styrene-sulfonic acid) (PEDOT:PSS) [15], and silicon nanowires [16] have been investigated. Despite the contribution to sensing performance improvement, the usage of sensing materials inevitably increases the production cost and fabrication complexity of the sensors. Meanwhile, the effective RH sensing ranges of these sensors may also be limited by the sensing materials, which hinders their practical applications to some extent. Therefore, remarkable efforts have been devoted to develop RH sensors using novel, passive microwave structures without sensing materials [22-26].

Among various topologies, substrate integrated waveguide (SIW) has gained increasing attention due to its low cost, low profile, high power capacity, and easy integration with other planar circuits. An SIW cavity available for RH sensing was first presented by Matbouly et al. [22]. Thereafter, a modified air-filled SIW cavity was proposed with the aim of improving sensitivity [23]. Through fully replacing the middle substrate of the cavity with moist air, a sensitivity up to $1.21 \mathrm{MHz} / \% \mathrm{RH}$ (with an operating frequency of $7.63 \mathrm{GHz}$ ) was successfully realized. Nevertheless, the overall sizes of these structures are too large, making them unsuitable for compact integrated systems. As an attempt to address this issue, Jones et al. proposed a ridged quarter-mode SIW (QMSIW) humidity sensor [24]. By introducing the ridged quarter-mode technique, it achieved a size reduction of $86.2 \%$ compared to the sensor reported in [22]. However, this design suffers from a poor sensitivity (only $30.73 \mathrm{kHz} / \% \mathrm{RH}$ at $6.9 \mathrm{GHz}$ ), and its loaded Q-factor is also relatively low due to the open boundaries inherent with quarter-mode structures. From these reported works, one can see that the realization of SIW humidity sensors simultaneously possessing high sensing performance and miniaturized size still remains a challenge.

Herein, we propose a novel double-folded SIW (DFSIW) re-entrant cavity and demonstrate its ability for highly sensitive humidity sensing both numerically and experimentally. The overall size of the cavity is dramatically miniaturized by combining the folding technology with re-entrant configuration. Numerical analyses show that it only occupies $14.1 \%$ of the overall size of conventional $\mathrm{TM}_{010}$-mode SIW circular cavities. The high sensitivity property of the sensor is achieved by introducing sensitive regions inside the cavity where the electric field is highly concentrated. When moist air enters the sensitive regions, it causes a strong perturbation to the electric field and results in a large resonant frequency shift of the cavity. This structural design offers an effective solution for balancing the sensing performance and physical dimensions of a passive microwave humidity sensor, which is of great significance for promoting the development and application of microwave humidity sensing devices.

\section{Sensing Mechanism and Sensor Design}

\subsection{Sensing Mechanism}

The working principle of the designed DFSIW re-entrant cavity humidity sensor is based on the cavity perturbation method. The moist air can be regarded as a dielectric medium, whose dielectric constant is related to $R H$ (unit: \%) as follows [3,22]:

$$
\varepsilon_{r}(R H)=1+\frac{211}{T}\left(P+48 \frac{P_{s}}{T} R H\right) 10^{-6},
$$

where $T$ is the absolute temperature (unit: K). $P$ and $P_{S}$ represent the pressures of moist air and saturated water vapor (unit: $\mathrm{mmHg}$ ), respectively.

When the cavity is exposed to a humid environment, the moist air spreads into the sensitive region of the cavity, and then causes perturbation to the induced electric field of the cavity, finally leading 
to a remarkable change in the resonant frequency. According to the cavity-material perturbation theory $[27,28]$, the fractional frequency shift can be approximately computed as:

$$
\frac{f_{r}-f_{0}}{f_{0}} \approx-\frac{\int_{V_{0}}\left(\Delta \varepsilon \boldsymbol{E} \cdot \boldsymbol{E}_{0}^{*}+\Delta \mu \boldsymbol{H} \cdot \boldsymbol{H}_{0}^{*}\right) d v}{\int_{V_{0}}\left(\varepsilon\left|\boldsymbol{E}_{0}\right|^{2}+\mu\left|\boldsymbol{H}_{0}\right|^{2}\right) d v},
$$

where $f_{r}$ and $f_{0}$ represent the resonant frequency of the perturbed and unperturbed cavities, respectively. $E(H)$ and $E_{0}\left(H_{0}\right)$ are the induced electric field (magnetic field) of the cavity with and without perturbation, respectively, while $\boldsymbol{E}_{0}^{*}$ and $\boldsymbol{H}_{0}^{*}$ are the complex conjugates of $\boldsymbol{E}_{0}$ and $\boldsymbol{H}_{0}$, respectively. $V_{0}$ represents the volume of the cavity. $\Delta \varepsilon$ and $\Delta \mu$ denote the variation in permittivity and permeability introduced by the air sample, respectively. As aforementioned, the moist air is considered as a dielectric medium, so the variation in permeability should be equal to zero (i.e., $\Delta \mu=0$ ). Then, by using a quasi-static approximation of the field internal to the air sample [29], Equation (2) can be further simplified to

$$
\frac{f_{r}-f_{0}}{f_{0}} \approx-\frac{\int_{V_{s}} \Delta \varepsilon \boldsymbol{E}_{i n t} \cdot \boldsymbol{E}_{0}^{*} d v}{2 \int_{V_{0}} \varepsilon\left|\boldsymbol{E}_{0}\right|^{2} d v},
$$

where $\boldsymbol{E}_{\text {int }}$ is the electric field internal to the moist air at the sensitive region and $V_{s}$ is the effective volume occupied by the sample. The internal electric field $\boldsymbol{E}_{i n t}$ is formed by the polarization effect of the air sample under the excitation of $E_{0}$, and it is mainly determined by the shape of the sample and the relative orientation between the sample and $E_{0}$. As for the designed sensor in this work, the shape of the air sample can be modeled as a thin circular sector slab with $E_{0}$ normal to it. For such a configuration, the $\boldsymbol{E}_{\text {int }}$ associating with $\boldsymbol{E}_{0}$ is given as [29]

$$
\boldsymbol{E}_{\text {int }}=\frac{1}{\varepsilon_{r}(R H)} \boldsymbol{E}_{0}
$$

Substituting Equation (4) into Equation (3), we finally obtain

$$
\frac{f_{r}-f_{0}}{f_{0}} \approx-\frac{1}{2} A \times \frac{\varepsilon_{r}(R H)-1}{\varepsilon_{r}(R H)},
$$

where $A=\frac{\int_{V_{s}}\left|E_{0}\right|^{2} d v}{\int_{V_{0}}\left|E_{0}\right|^{2} d v}$, and it is mainly affected by the volume ratio of the air sample to the cavity. Given that $V_{s}$ is always smaller than $V_{0}$, the value of $A$ should be in the range of $0 \leq A<1$. Once the geometrical parameters of the cavity are fixed, $A$ would be kept as a constant. Its value can be determined accurately using a calibration method. The above theoretical model shows that the fractional frequency shift of the sensor is a function of the RH level. That is, the RH condition of the ambient air can be easily retrieved by measuring the corresponding resonant frequency variation of the sensor.

\subsection{DFSIW Re-Entrant Cavity Design}

Figure 1 illustrates the geometrical structure of the proposed DFSIW re-entrant cavity humidity sensor, which is generated by folding a conventional circular re-entrant cavity twice along its two orthogonally symmetric planes. The structure consists of four dielectric substrate layers sandwiched between multiple metallic layers. The dielectric substrate used was F4B-2 with a dielectric constant of 2.65 and a loss tangent of 0.001 , and the metallic layer was a copper film with a thickness of $35 \mu \mathrm{m}$. An L-shaped slot was etched on the bottom metallic layer of substrate 2 and the top metallic layer of substrate 3. This slot is the key element for realizing folded structures [30-32]. Its main function is to create fictitious magnetic walls that enable the propagation of electromagnetic waves from the upper substrates to the lower ones. In this manner, the desired electromagnetic field distribution 
in a folded form can be obtained. By choosing an appropriate value of the slot width, the folded cavity is able to achieve the similar resonant properties to its unfolded counterpart [33]. The sensitive regions for humidity detection were introduced in substrates 2 and 3, with the same area as the capacitive post. To allow the entrance of moist air into the cavity, two orthogonal rows of air holes were drilled on substrates 1 and 4 . The ambient sidewalls of the cavity were created through a number of metallized vias that connect all the metallic layers. Note that the diameter of the via $\left(d_{\mathrm{vc}}\right)$ and the spacing between two adjacent vias $\left(s_{\mathrm{vc}}\right)$ should obey the constraint of $\frac{s_{\mathrm{vc}}}{2} \leq d_{\mathrm{vc}}<\frac{\lambda_{g}}{5}$, with $\lambda_{g}$ being the guided wavelength. This is to ensure that the radiation loss between adjacent vias can be neglected. Specifically, $d_{\mathrm{vc}}=1 \mathrm{~mm}$ and $s_{\mathrm{Vc}}=1.4 \mathrm{~mm}$ were used in this design. The excitation of the cavity was accomplished using a tapered coplanar waveguide (CPW) feedline with a characteristic impedance of $50 \Omega$. The geometrical parameters of the final optimized cavity are listed in Table 1.
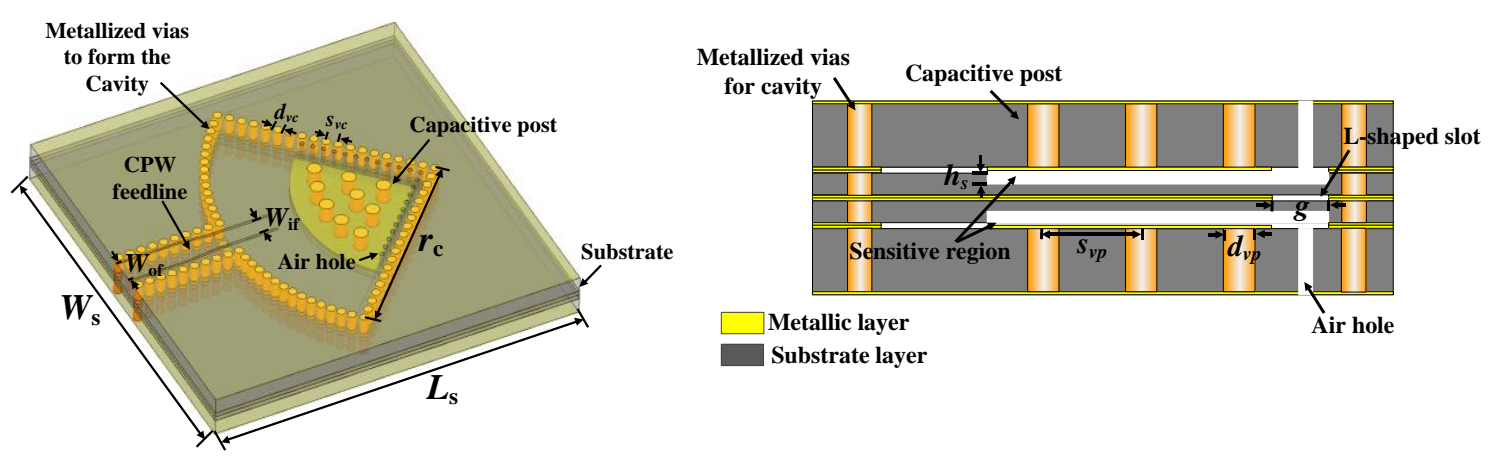

(a)

(b)

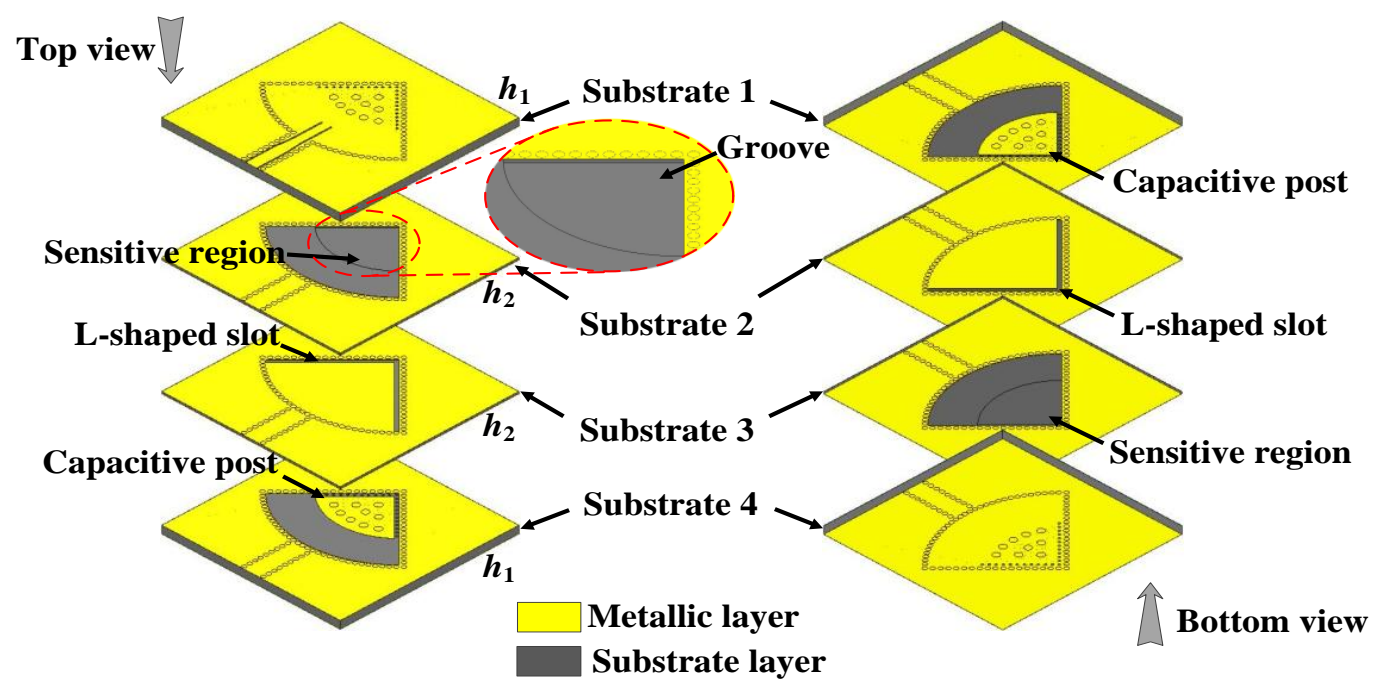

(c)

Figure 1. Diagram of the proposed double-folded substrate integrated waveguide (DFSIW) re-entrant cavity: (a) full view; (b) cutaway view (without feedline); (c) exploded view. 
Table 1. Geometrical parameters of the final optimized cavity.

\begin{tabular}{ccc}
\hline Parameter & Description & Value (mm) \\
\hline$L_{\mathrm{S}}$ & Total length of substrate & 45 \\
$W_{\mathrm{s}}$ & Total width of substrate & 45 \\
$L_{\mathrm{f}}$ & Length of CPW feedline* & 18.5 \\
$W_{\mathrm{of}}$ & Outer width of CPW feedline & 2.77 \\
$W_{\mathrm{if}}$ & Inner width of CPW feedline & 2 \\
$r_{\mathrm{c}}$ & Radius of cavity & 25.2 \\
$r_{\mathrm{p}}$ & Radius of capacitive post & 14.1 \\
$d_{\mathrm{vp}}$ & Diameter of via of capacitive post & 1.6 \\
$s_{\mathrm{vp}}$ & Space between two adjacent vias of capacitive post & 3.31 \\
$d_{\mathrm{ah}}$ & Diameter of air hole & 0.5 \\
$s_{\mathrm{ah}}$ & Space between two adjacent air holes & 1.25 \\
$g$ & Width of L-shaped slot & 0.9 \\
$h_{1}$ & Height of substrates 1 and 4 & 2 \\
$h_{2}$ & Height of substrates 2 and 3 & 0.5 \\
$h_{\mathrm{s}}$ & Height of sensitive region & 0.3 \\
\hline & $*$ CPW coplanar waveguide.
\end{tabular}

To quantitatively demonstrate the ability of the designed structure for size miniaturization, the resonant properties of three kinds of cavities (conventional circular SIW cavity, unfolded SIW re-entrant cavity, our proposed DFSIW re-entrant cavity) were investigated using the Finite Difference Time Domain (FDTD) method. Figure 2 shows the simulated reflection coefficients $S_{11}$ of these three cavities, as well as their electric field distributions at resonances. For better comparisons, the resonant cavity areas and heights of these three cavities were set to be identical (158.76 $\left.\pi \mathrm{mm}^{2} \times 5 \mathrm{~mm}\right)$. From Figure 2, the circular SIW cavity operating in the $\mathrm{TM}_{010}$ mode resonated at $5.79 \mathrm{GHz}$, while the re-entrant cavity exhibited a lower resonant frequency of $3.22 \mathrm{GHz}$. This frequency shift, corresponding to a relative size reduction of $70.1 \%$, is mainly attributed to the large equivalent capacitance introduced by the capacitive post of the re-entrant cavity [34]. Taking a further step, the re-entrant cavity was folded twice along its two orthogonally symmetric planes, forming the so-called DFSIW re-entrant cavity. Due to this folding operation, the resonant frequency of the cavity was further decreased to $2.18 \mathrm{GHz}$, which means that a further miniaturization of $54.2 \%$ was realized. It is emphasized once again that the resonant cavity area of the DFSIW re-entrant cavity was scaled up to $158.76 \pi \mathrm{mm}^{2}$ to keep it identical with the cavity area of the unfolded re-entrant cavity, and the size miniaturization considered here is for the electrical size (i.e., relative size with respect to the resonant frequency). If its cavity area was not scaled, the DFSIW re-entrant cavity would have the same resonant frequency with the unfolded re-entrant cavity. This can also be demonstrated by the electric field distributions of the cavities (shown in the insets of Figure 2). As can be observed, the DFSIW re-entrant cavity has an analogous but folded electric field distribution to that of the unfolded re-entrant cavity. This means that it can obtain the same resonant frequency as the unfolded re-entrant cavity while using only one quarter of the footprints [30]. Consequently, it can be concluded that the proposed DFSIW re-entrant cavity is very compact in size and hence can be applied to highly integrated systems. 


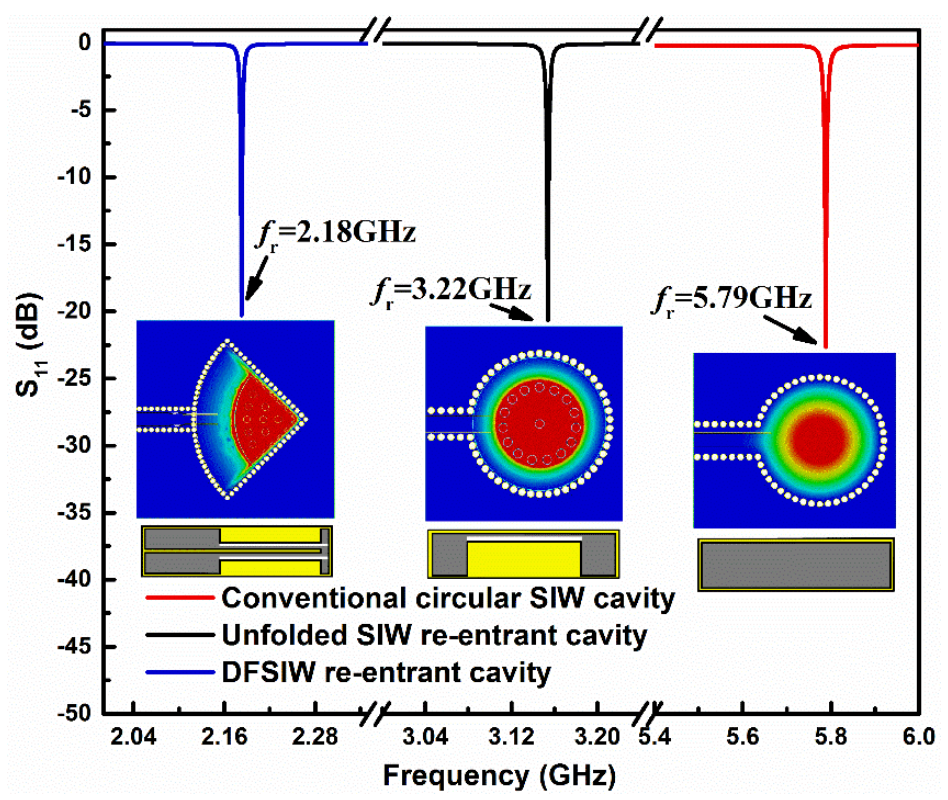

Figure 2. Simulated reflection coefficients $S_{11}$ of a conventional circular SIW cavity, an unfolded SIW re-entrant cavity, and the proposed DFSIW re-entrant cavity. Note that the resonant cavity areas of these three cavities were kept identical for a better comparison. The insets are the corresponding electric distributions of these three cavities at resonances.

\subsection{Parameter Analysis}

In order to further investigate the influence of the geometrical parameter of the sensitive region on the performance of the sensor, the resonant frequency and normalized sensitivity of the sensor with different heights of the sensitive region $h_{\mathrm{s}}$ were simulated, and the corresponding results are shown in Figure $3 \mathrm{a}, \mathrm{b}$, respectively. The normalized sensitivity was computed using the following formulation:

$$
S_{n}=\left|\frac{\left[f_{r}\left(R \boldsymbol{H}_{1}\right)-f_{r}\left(R \boldsymbol{H}_{2}\right)\right] / f_{r}\left(R \boldsymbol{H}_{1}\right)}{R \boldsymbol{H}_{1}-R \boldsymbol{H}_{2}}\right|,
$$

where $R H_{1}$ and $R H_{2}$ represent two different $\mathrm{RH}$ level (in \%), and $f_{r}\left(R \boldsymbol{H}_{1}\right)$ and $f_{r}\left(R \boldsymbol{H}_{2}\right)$ are the corresponding resonant frequencies for these two RH conditions, respectively. It is apparent from Figure 3a that the resonant frequency of the sensor was monotonically reduced with the decrease of $h_{\mathrm{s}}$. This suggests that the size miniaturization of the sensor can be further enhanced by setting a smaller $h_{\mathrm{s}}$. However, the decreasing $h_{\mathrm{s}}$ also contributed to the deterioration of the normalized sensitivity of the sensor (as shown in Figure 3b). This is due to the fact that the volume ratio of the sensitive region (or air sample) to the cavity is lowered and only limited perturbation to the original electric field can be obtained [35]. Therefore, through choosing a different value of $h_{\mathrm{s}}$, the designed sensor can be flexibly adjusted to have a more compact size or a higher sensitivity. This choice can be made according to the practical requirements. In the presented paper, the value of $h_{\mathrm{s}}$ was set to be $0.3 \mathrm{~mm}$, so as to balance the relative size and sensing performance of the sensor. 


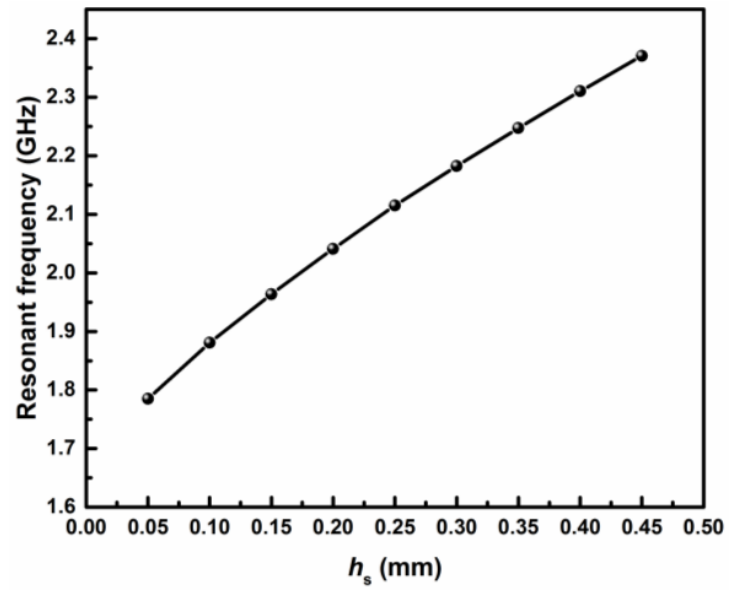

(a)

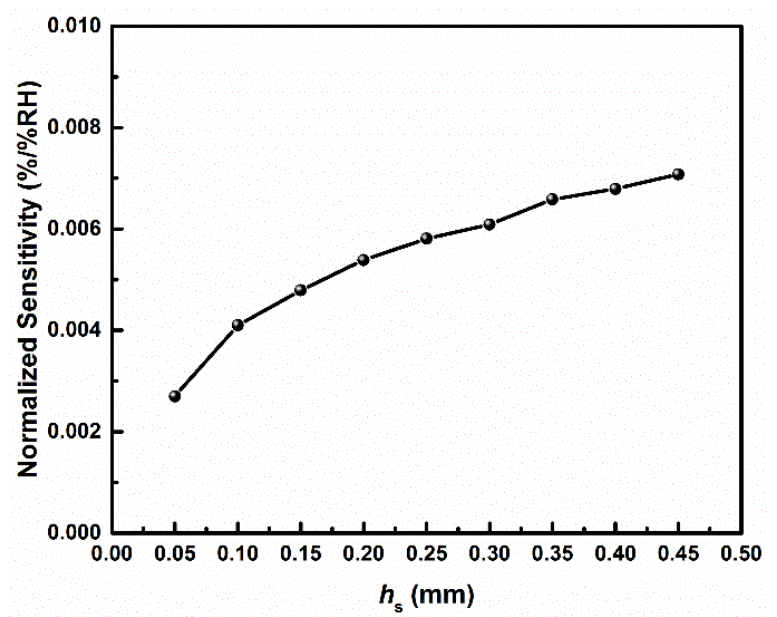

(b)

Figure 3. Simulated (a) resonant frequency and (b) normalized sensitivity of the sensor with varying heights of the sensitive region, $h_{\mathrm{s}}$.

\section{Fabrication, Measurement, and Discussion}

\subsection{Sensor Fabrication and Measurement Setup}

To experimentally demonstrate its sensing performance, the proposed DFSIW re-entrant cavity humidity sensor was fabricated using standard printed circuit board (PCB) technology. The four layers (shown in Figure 1c) were separately manufactured in the fabrication process. Both the capacitive posts on substrates 1 and 4 were built using a copper plate together with a metallized vias array embedded in the substrate. The sensitive regions were created by etching two identical circular sector grooves on the top surface of substrate 2 and the bottom surface of substrate 3. Each layer was drilled with twelve screw holes so as to align and tighten all the layers together. Finally, an SMA (sub-miniature version A) connector was soldered to the sensor for measurement purposes. The photographs of the fabricated sensor prototype, including the top views of each layer and the finally assembled sensor, are presented in Figure 4.

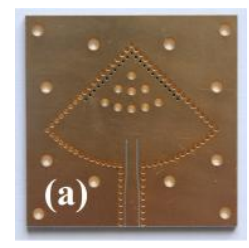

(a)

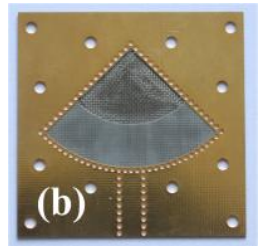

(b)

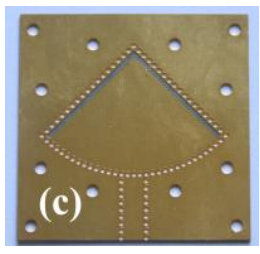

(c)

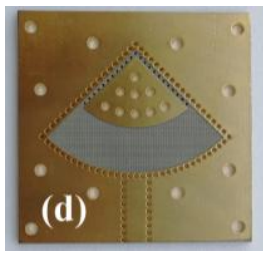

(d)

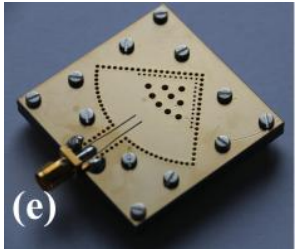

(e)

Figure 4. The photographs of the fabricated sensor prototype: (a) first layer, (b) second layer, (c) third layer, (d) forth layer, and (e) the final assembled sensor.

Figure 5 depicts the experimental setup for the fabricated humidity sensor. The sensor was placed inside a humidity chamber (KW-THD-208X, KOWINTEST EQUIPMENT, Dongguan, China), where the $\mathrm{RH}$ level was varied from $30 \%$ to $80 \%$ with a step size of $10 \%$. To extract the required resonant frequency, the reflection coefficient $S_{11}$ of the sensor was measured using a vector network analyzer (VNA) (E5071C, Agilent, Beijing, China). Before the measurement, the VNA was calibrated using a standard calibration kit 85052D. In order to verify the repeatability of the sensor, the measurement for each RH condition was repeated at least four times, and the final results are the averaged values of multiple measurements. 


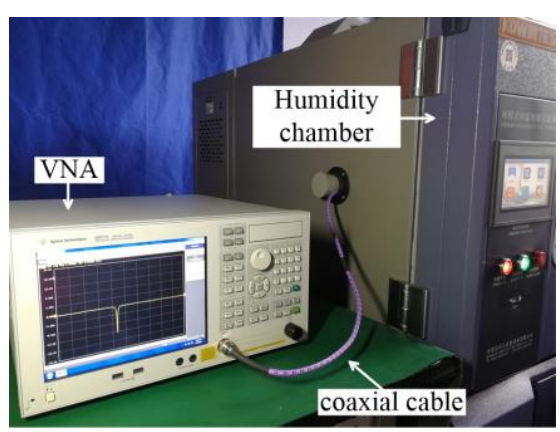

(a)

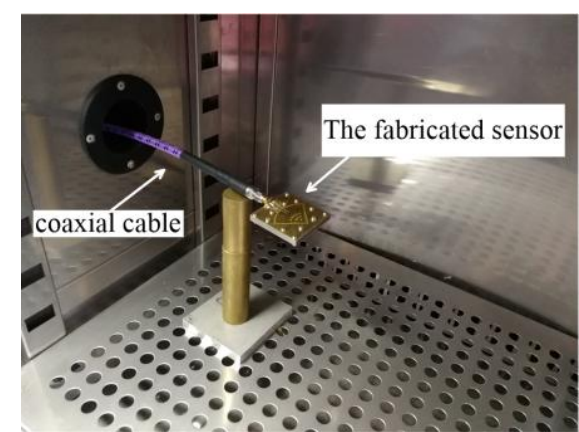

(b)

Figure 5. Photographs of the measurement setup for the designed DFSIW re-entrant cavity humidity sensor: (a) full measurement setup; (b) setup inside the humidity chamber.

\subsection{Result and Discussion}

Figure 6 shows the simulated and measured $\mathrm{S}_{11}$ of the designed humidity sensor at $30 \% \mathrm{RH}$. It can be seen that the measured results are in good agreement with the simulated ones except for a tiny frequency deviation and a minor decrease in return loss. The frequency deviation could be mainly attributed to the imprecise dielectric constant of the actual substrate or the fabrication tolerance (especially the tolerance on the height of the sensitive region $h_{\mathrm{s}}$ ). The amplitude decrease may be caused by the losses incurred from the substrate or the mismatch between the SMA connector and the cavity. The measured loaded Q-factor of the sensor was calculated to be 266. Such a high Q-factor, corresponding to a sharp resonant peak, makes it easier to trace the resonant frequency shift for small variations of $\mathrm{RH}$ and, therefore, improves the resolution of the sensor.

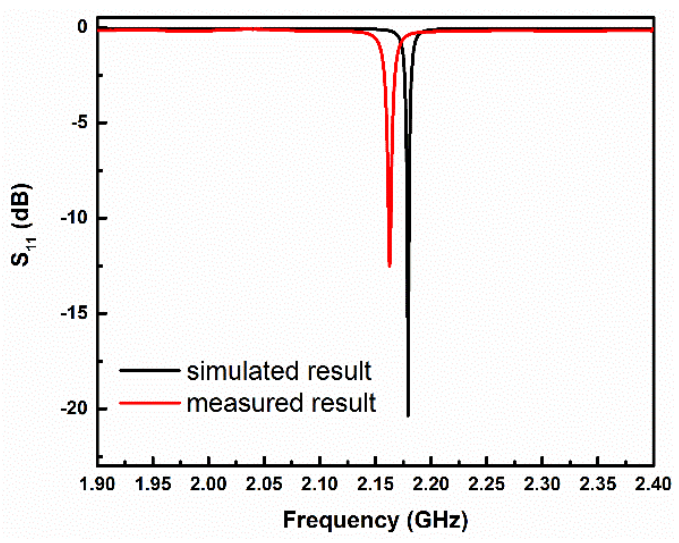

Figure 6. Simulated and measured reflection coefficients $\mathrm{S}_{11}$ of the designed humidity sensor at $30 \%$ relative humidity $(\mathrm{RH})$.

Figure 7 shows the measured $\mathrm{S}_{11}$ of the designed humidity sensor at different $\mathrm{RH}$ conditions. It is clear that the measured resonant frequency of the sensor exhibits a continuous redshift with the increase of RH. This phenomenon is in accordance with the sensing mechanism analyzed in Section 2.1. Namely, the relative permittivity of ambient air would become higher with the increase of $\mathrm{RH}$, resulting in a stronger perturbation to the induced electric field of the sensor and finally generating a larger frequency shift. Figure 8 plots the measured resonant frequency of the sensor as a function of the RH level, together with the calculated results using the theoretical model. The error bars denote the standard deviation of resonant frequency for the multiple, repeated measurements under each RH condition. As can be seen from Figure 8, when the RH level rises from $30 \%$ to $80 \%$, the measured resonant frequency of the sensor is monotonically reduced from $2.16279 \mathrm{GHz}$ to $2.15601 \mathrm{GHz}$, corresponding to an absolute frequency shift of $6.78 \mathrm{MHz}$. By using the calculation 
formula of $S_{a}=\frac{\Delta f}{\Delta \% R H}[22,24]$, the measured absolute sensitivity of our designed sensor was calculated to be $135.6 \mathrm{kHz} / \% \mathrm{RH}$. If we set the frequency step size of the VNA as $12.5 \mathrm{kHz}$ (e.g., frequency range from 2.15 to $2.17 \mathrm{GHz}$ with 1601 points), the resolution of the sensor is then given as $0.09 \% \mathrm{RH}$.

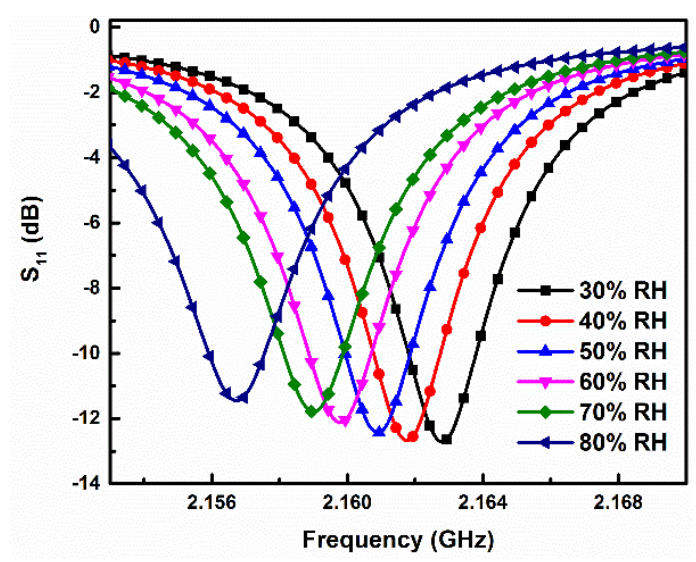

Figure 7. Measured reflection coefficients $S_{11}$ of the designed humidity sensor at different RH levels.

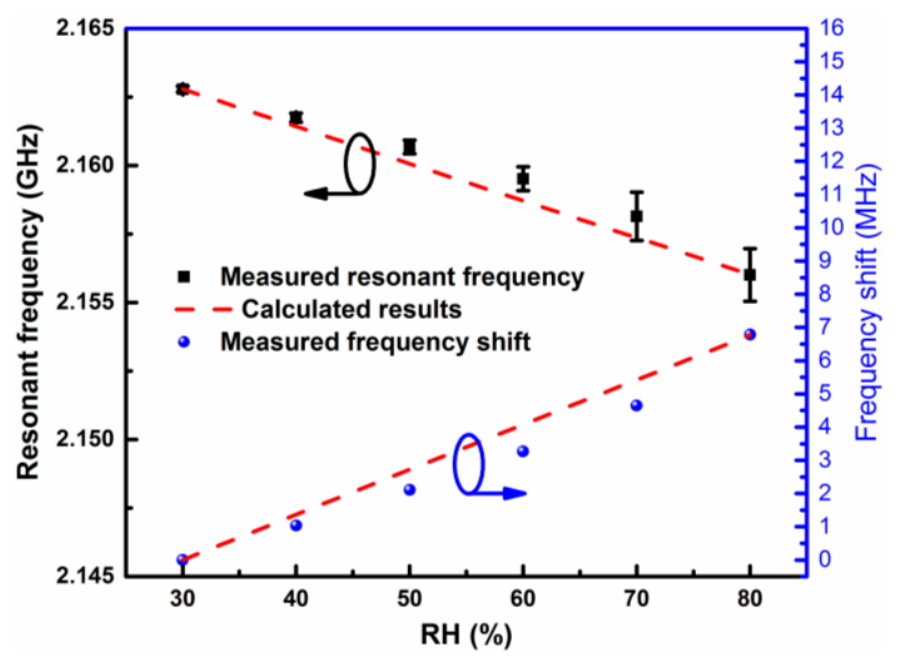

Figure 8. Measured resonant frequency of the designed sensor as a function of RH level.

Table 2 summarizes the comparisons of some key figure-of-merit between our proposed sensor and other recently reported passive microwave humidity sensors. Considering that the operating frequencies of these sensors are different from each other, the normalized sensitivity is used here to ensure fair comparisons. Analogously, the dimension of the sensor is also given as a relative size with respect to the wavelength $\lambda_{0}$. As indicated in Table 2, the air-filled SIW structure in reference [23] provides the highest sensitivity but it requires a large dimension. In contrast, the QMSIW cavity in reference [24] effectively reduces the size whereas its sensitivity is too low. In comparison to these reported state-of-the-art humidity sensors, the proposed one in this paper not only has a much smaller relative size, but also offers a very competitive sensitivity (only a little lower than the air-filled SIW cavity). The measured loaded Q-factor of the proposed sensor is also at a relatively satisfactory level. Additionally, its planar SIW structure also enables it to integrate with other planar circuits easily (without any costly and sophisticated transition needed [36]). These features make the proposed sensor a potential candidate for humidity sensing applications in various fields. 
Table 2. Comparison of other recently published works and this work.

\begin{tabular}{ccccccc}
\hline Ref. & Sensor Structure & $\begin{array}{c}\text { Operating } \\
\text { Frequency } \\
\text { (GHz) }\end{array}$ & Relative Size + & $\begin{array}{c}\text { Measured } \\
\text { Loaded } \\
\text { Q-Factor }\end{array}$ & $\begin{array}{c}\text { RH } \\
\text { Measurement } \\
\text { Range (\%) }\end{array}$ & $\begin{array}{c}\text { Normalized } \\
\text { Sensitivity } \\
(\% / \% R H)\end{array}$ \\
\hline$[22]-1$ & SIW cavity & 4.15 & $\left(0.43 \lambda_{0} \times 0.43 \lambda_{0}\right) \S$ & 263.25 & $0-80$ & $2.43 \times 10^{-3}$ \\
{$[22]-2$} & 3.6 & $0.373 \lambda_{0} \times 0.373 \lambda_{0}$ & 308 & $0.26 \times 10^{-3}$ \\
{$[23]$} & Air-filled SIW cavity & 7.63 & $0.712 \lambda_{0} \times 0.712 \lambda_{0}$ & 273.2 & $20-85$ & $15.8 \times 10^{-3}$ \\
{$[24]-1$} & & 6 & $0.236 \lambda_{0} \times 0.236 \lambda_{0}$ & 35 & $0-80$ & $0.608 \times 10^{-3}$ \\
{$[24]-2$} & QMSIW cavity & 6.9 & $0.178 \lambda_{0} \times 0.178 \lambda_{0}$ & 86 & $0-70$ & $0.445 \times 10^{-3}$ \\
{$[25]$} & SIW interferometer & 16.55 & $5.4 \lambda_{0} \times 2.54 \lambda_{0}$ & 2400 & $20-70$ & $0.858 \times 10^{-3}$ \\
This work & DFSIW re-entrant cavity & 2.163 & $0.161 \lambda_{0} \times 0.161 \lambda_{0}$ & 266 & $30-80$ & $6.27 \times 10^{-3}$ \\
\hline
\end{tabular}

\$QQMSIW = quarter-mode SIW; † Only the sizes of cavity areas are calculated; $\S \lambda_{0}$ represents the free space wavelength at operating frequency.

\section{Conclusions}

A novel DFSIW re-entrant cavity dedicated to humidity detection is presented in this paper. The concept of incorporating folding technology with re-entrant configuration is proposed and is proven to be an effective solution for realizing ultra-compact resonator design. Compared to the conventional circular SIW cavity, the proposed structure has achieved a size miniaturization up to $85.9 \%$. It also can be readily integrated with other planar circuits thanks to the SIW technology. The sensing mechanism of the humidity sensor was analyzed using cavity perturbation theory. It was found that the resonant frequency of the senor behaves as a function of the RH level. Consequently, the resonant frequency was employed as the signature of the sensor to monitor the RH condition of ambient air. A sensor prototype operating at $2.16 \mathrm{GHz}$ was fabricated and its sensing performance was experimentally tested. The measured results demonstrate that in the $\mathrm{RH}$ range of $30 \%-80 \%$, the designed sensor exhibits an absolute sensitivity of $135.6 \mathrm{kHz} / \% \mathrm{RH}$ and a relative sensitivity of $0.00627 \% / \% \mathrm{RH}$ (normalized to the operating frequency). The proposed humidity sensor, characterized by a compact size, low cost, high Q-factor, high sensitivity, and ease of fabrication and integration, is expected to find potential applications in environment monitoring or gas sensing.

Author Contributions: Conceptualization, Z.W. and J.H.; Data curation, Z.W. and X.L.; Funding acquisition, J.H.; Investigation, Z.W. and J.L. (Jing Li); Supervision, J.H.; Validation, J.H. and X.N.; Writing—original draft, Z.W. and J.L. (Junshan Li); Writing_-review and editing, J.H. and J.L. (Jing Li).

Funding: This research was supported in part by the Young Scientists Fund of the National Natural Science Foundation of China [Grant No. 61401373], in part by the Fundamental Research Funds for the Central Universities [Grant No. XDJK2018B017 and Grant No. XDJK2019D016], and in part by the Research Fund for the Doctoral Program of Southwest University [Grant No. SWU111030].

Conflicts of Interest: The authors declare no conflict of interest.

\section{References}

1. Grange, R.I.; Hand, D.W. A review of the effects of atmospheric humidity on the growth of horticultural crops. J. Hortic. Sci. Biotech. 1987, 62, 125-134. [CrossRef]

2. Tu, K.; Nicolai, B.; De Baerdemaeker, J. Effects of relative humidity on apple quality under simulated shelf temperature storage. Sci. Hortic. 2000, 85, 217-229. [CrossRef]

3. Jacob, F. Handbook of Modern Sensors: Physics, Designs, and Applications, 4th ed.; Springer: New York, NY, USA, 2010.

4. Chen, Z.; Lu, C. Humidity sensors: A review of materials and mechanisms. Sens. Lett. 2005, 3, 274-295. [CrossRef]

5. Su, P.-G.; Wang, C.-S. Novel flexible resistive-type humidity sensor. Sens. Actuators B Chem. 2007, 123, 1071-1076. [CrossRef]

6. Lim, D.-I.; Cha, J.-R.; Gong, M.-S. Preparation of flexible resistive micro-humidity sensors and their humidity-sensing properties. Sens. Actuators B Chem. 2013, 183, 574-582. [CrossRef]

7. Zhang, H.Y.; Zhang, M.; Lin, C.C.; Zhang, J. AuNPs Hybrid Black ZnO Nanorods Made by a Sol-Gel Method for Highly Sensitive Humidity Sensing. Sensors 2018, 18, 218. [CrossRef] 
8. Zhang, D.Z.; Chang, H.Y.; Li, P.; Liu, R.H.; Xue, Q.Z. Fabrication and characterization of an ultrasensitive humidity sensor based on metal oxide/graphene hybrid nanocomposite. Sens. Actuators B Chem. 2016, 225, 233-240. [CrossRef]

9. Chung, V.P.J.; Yip, M.-C.; Fang, W.L. Resorcinol-formaldehyde aerogels for CMOS-MEMS capacitive humidity sensor. Sens. Actuators B Chem. 2015, 214, 181-188. [CrossRef]

10. Tripathy, A.; Pramanik, S.; Manna, A.; Bhuyan, S.; Shah, N.F.A.; Radzi, Z.; Osman, N.A.A. Design and Development for Capacitive Humidity Sensor Applications of Lead-Free Ca,Mg,Fe,Ti-Oxides-Based Electro-Ceramics with Improved Sensing Properties via Physisorption. Sensors 2016, 16, 1135. [CrossRef]

11. Ren, X.N.; Zhang, D.Z.; Wang, D.Y.; Li, Z.G.; Liu, S.X. Quartz Crystal Microbalance Sensor for Humidity Sensing Based on Layer-by-Layer Self-Assembled PDDAC/Graphene Oxide Film. IEEE Sens. J. 2018, 18, 9471-9476. [CrossRef]

12. Chen, X.P.; Chen, X.D.; Li, N.; Ding, X.; Zhao, X. A QCM Humidity Sensors Based on GO/Nafion Composite Films with Enhanced Sensitivity. IEEE Sens. J. 2016, 16, 8874-8883. [CrossRef]

13. Lao, C.S.; Kuang, Q.; Wang, Z.L.; Park, M.-C.; Deng, Y.L. Polymer functionalized piezoelectric-FET as humidity/chemical nanosensors. Appl. Phys. Lett. 2007, 90, 262107. [CrossRef]

14. Amin, E.M.; Karmakar, N.C.; Winther-Jensen, B. Polyvinyl-alcohol (PVA)-based RF humidity sensor in microwave frequency. Prog. Electromagn. Res. B 2013, 54, 149-166. [CrossRef]

15. Manzari, S.; Occhiuzzi, C.; Nawale, S.; Catini, A.; Di Natale, C.; Marrocco, G. Humidity sensing by polymer-loaded UHF RFID antennas. IEEE Sens. J. 2012, 12, 2851-2858. [CrossRef]

16. Nair, R.; Perret, E.; Tedjini, S.; Barron, T. A humidity sensor for passive chipless RFID applications. In Proceedings of the IEEE International Conference on RFID-Technologies and Applications (RFID-TA), Nice, France, 5-7 November 2012; pp. 29-33. [CrossRef]

17. Chen, C.M.; Xu, J.; Yao, Y. Fabrication of miniaturized CSRR-loaded HMSIW humidity sensors with high sensitivity and ultra-low humidity hysteresis. Sens. Actuators B Chem. 2018, 256, 1100-1106. [CrossRef]

18. Bogner, A.; Steiner, C.; Walter, S.; Kita, J.; Hagen, G.; Moos, R. Planar Microstrip Ring Resonators for Microwave-Based Gas Sensing: Design Aspects and Initial Transducers for Humidity and Ammonia Sensing. Sensors 2017, 17, 2422. [CrossRef] [PubMed]

19. Chen, C.M.; Xu, J.; Yao, Y. SIW resonator humidity sensor based on layered black phosphorus. Electron Lett. 2017, 53, 249-251. [CrossRef]

20. Tan, Q.L.; Lv, W.; Ji, Y.H.; Song, R.J.; Lu, F.; Dong, H.L.; Zhang, W.D.; Xiong, J.J. A LC wireless passive temperature-pressure-humidity (TPH) sensorintegrated on LTCC ceramic for harsh monitoring. Sens. Actuators B Chem. 2018, 270, 433-442. [CrossRef]

21. Eyebe, G.A.; Bideau, B.; Boubekeur, N.; Loranger, E.; Domingue, F. Environmentally-friendly cellulose nanofibre sheets for humidity sensing in microwave frequencies. Sens. Actuators B Chem. 2017, 245, 484-492. [CrossRef]

22. El Matbouly, H.; Boubekeur, N.; Domingue, F. Passive microwave substrate integrated cavity resonator for humidity sensing. IEEE Trans. Microw. Theory Tech. 2015, 63, 4150-4156. [CrossRef]

23. Ndoye, M.; Kerroum, I.; Deslandes, D.; Domingue, F. Air-filled substrate integrated cavity resonator for humidity sensing. Sens. Actuators B Chem. 2017, 252, 951-955. [CrossRef]

24. Jones, T.R.; Zarifi, M.H.; Daneshamand, M. Miniaturized Quarter-Mode Substrate Integrated Cavity Resonators for Humidity Sensing. IEEE Microw. Wirel. Compon. Lett. 2017, 27, 612-614. [CrossRef]

25. Benleulmi, A.; Boubekeur, N.; Massicotte, D. A Highly Sensitive Substrate Integrated Waveguide Interferometer Applied to Humidity Sensing. IEEE Microw. Wirel. Compon. Lett. 2018, 29, 68-70. [CrossRef]

26. Ho, A.T.; Ndoye, M.; Ferrari, P.; Domingue, F. Miniaturized Humidity Sensor based on a partially Air-filled Slow-Wave SIW Resonator. In Proceedings of the 48th European Microwave Conference (EuMC2018), Madrid, Spain, 23-27 September 2018; pp. 25-27. [CrossRef]

27. Chen, L.F.; Ong, C.K.; Neo, C.P.; Varadan, V.V.; Varadan, V.K. Microwave Electronics: Measurement and Materials Characterization; John Wiley \& Sons: Chichester, West Sussex, England, 2004. [CrossRef]

28. Pozar, D.M. Microwave Engineering, 3rd ed.; John Wiley \& Sons: New York, NY, USA, 2005.

29. Harrington, R.F. Time Harmonic Electromagnetic Fields; McGraw-Hill: New York, NY, USA, 1961.

30. Hong, J.-S. Compact folded-waveguide resonators and filters. IEEE Proc.-Microw. Antennas Propag. 2006, 153, 325-329. [CrossRef] 
31. Chien, H.-Y.; Shen, T.-M.; Huang, T.-Y.; Wang, W.-H.; Wu, R.-B. Miniaturized Bandpass Filters with Double-Folded Substrate Integrated Waveguide Resonators in LTCC and filters. IEEE Trans. Microw. Theory Tech. 2009, 57, 1774-1782. [CrossRef]

32. Chen, M.-Y.; Hong, W.C.; Ho, M.-H. Differential-Mode BPF Design Using Folded Substrate Integrated Waveguide Cavities. In Proceedings of the 2015 European Microwave Conference (EuMC), Paris, France, 7-10 September 2015; pp. 1030-1032. [CrossRef]

33. Grigoropoulos, N.; Sanz-Izquierdo, B.; Young, P.R. Substrate Integrated Folded Waveguides (SIFW) and Filters. IEEE Microw. Wirel. Compon. Lett. 2005, 15, 829-831. [CrossRef]

34. Wei, Z.H.; Huang, J.; Li, J.; Xu, G.Q.; Ju, Z.D.; Liu, X.Y.; Ni, X.S. A High-Sensitivity Microfluidic Sensor Based on a Substrate Integrated Waveguide Re-Entrant Cavity for Complex Permittivity Measurement of Liquids. Sensors 2018, 18, 4005. [CrossRef]

35. Rowe, D.J.; Al-Malki, S.; Abduljabar, A.A.; Porch, A.; Barrow, D.A.; Allender, C.J. Improved split-ring resonator for microfluidic sensing. IEEE Trans. Microw. Theory Tech. 2014, 62, 689-699. [CrossRef]

36. Germain, S.; Deslandes, D.; Wu, K. Development of substrate integrated waveguide power dividers. In Proceedings of the CCECE 2003-Canadian Conference on Electrical and Computer Engineering, Montreal, QC, Canada, 4-7 May 2003; pp. 1921-1924. [CrossRef]

(C) 2019 by the authors. Licensee MDPI, Basel, Switzerland. This article is an open access article distributed under the terms and conditions of the Creative Commons Attribution (CC BY) license (http://creativecommons.org/licenses/by/4.0/). 